\title{
INCIDENCE OF BREAST LYMIPHEDEMA AND PREDICTORS OF ITS DEVELOPMENT IN PATIENTS RECEIVING WHOLE BREAST RADIATION THERAPY AFTER BREAST-CONSERVATION SURGERY
}

\author{
R.G. Ganju, G. Savvides, S. Korentager, M.J. Ward, \\ M. TenNapel, A. Amin, J. Wagner, M. Mitchell
}

Departments of Radiation Oncology (RGG,GS,MTN) and Surgery (SK,MJW,AA,JW), University of Kansas School of Medicine, Kansas City, Kansas and Department of Radiation Oncology (MM), University of Texas, MD Anderson Cancer Center, Houston, Texas USA

\section{ABSTRACT}

One common adverse effect following breast-conservation surgery and adjuvant radiation is lymphedema. While lymphedema of the arm has been well-characterized, there has been less investigation into lymphedema of the breast. We sought to characterize rates of breast lymphedema (BLE) in women with early-stage breast cancer and identify potential predictors in its development. Two hundred and thirty consecutive patients treated with lumpectomy and adjuvant whole breast radiation therapy (WBRT) from January 2016 - June 2017 were included. All patients were seen in our lymphedema monitoring clinic for baseline and at least one follow-up lymphedema measurement. BLE grades were assigned by trained nurses in the lymphedema clinic. Data regarding patient demographic and treatment factors were extracted from the electronic medical record. Comparisons between groups were made using Chi-Square analysis performed in $S A S$. The median age of the sample was 62 (range 31-90). Median follow-up from surgery was 15.3 months. Fortythree patients were diagnosed with lymphedema of the breast (18.7\%). Rates of grade 1 and 2 $B L E$ were $93 \%$ and $7 \%$, respectively; there were no cases of severe lymphedema. Sixty-three percent of cases resolved by last follow-up with treatment recommendations. There was no association between development of BLE and patient factors investigated, including age, $T$ stage, radiation dose and fractionation, lymph node biopsy, number of lymph nodes removed, development of arm lymphedema, and use of chemotherapy. Tumor subtype was found to be significant $(P=0.04)$ and there was a trend towards significance for receipt of trastuzumab $(P=0.09)$. BLE is a distinct entity from arm lymphedema and is a common finding in women treated with breast-conserving surgery and adjuvant WBRT. It is a generally mild and self-limiting process. There were no treatment or patient-related factors that correlated with increased risk of lymphedema development in our sample except for HER-2 positive disease and receipt of trastuzamab.

Keywords: breast-conservation surgery, breast lymphedema, adjuvant whole breast irradiation, predictors

Adjuvant whole breast irradiation in women who undergo lumpectomy has been well-established to decrease the risk of locoregional recurrence and improve survival in women with early stage breast cancer (1). As survival has improved in this patient population (2), there has been an emphasis towards better characterizing and minimizing adverse effects of treatment, and improving quality of life. As a result, modern treatment paradigms 
generally involve less invasive surgical techniques and utilize hypofractionated radiation regimens. Some studies suggest that these newer techniques for local treatment may impact toxicity and patient quality of life.

While lymphedema of the arm is a wellknown toxicity for breast cancer survivors, a common symptom after radiation, lymphedema of the breast, has been less well defined. Clinically, breast lymphedema is generally accepted to consist of persistent skin edema and erythema of the involved breast as well as breast heaviness or swelling (3). Onset is usually several months after the completion of radiation therapy. Development of breast lymphedema is known to result in decreased quality of life and can result in increases in distress $(3,4)$. It increases the risk of infection and may impair wound healing. We recently implemented a dedicated nurse-run lymphedema monitoring and prevention clinic for all patients undergoing surgery at our institution to assess both arm and breast lymphedema. We sought to characterize rates of breast lymphedema in patients with early-stage breast cancer who received lumpectomy and adjuvant radiation, as well as identify potential predictors in its development. Emphasis was placed on assessing the effect of total radiation dose and fractionation on the breast lymphedema endpoint.

\section{MATERIALS/METHODS}

\section{Patient Data}

Institutional review board approval was obtained for this retrospective review. We obtained the medical records of all patients with breast cancer undergoing lumpectomy followed by radiation from January 2016 through June 2017 at a single institution, including a central hospital and four satellite affiliated centers. Patients with a history of complete axillary lymph node dissection were excluded due to concerns that aggressive dissection may impact breast lymphedema. Most patients were early stage node negative; however, the sample population included a small number of patients with a low axillary burden who received radiation to the breast alone without dose to the supraclavicular or internal mammary nodes. Male patients and patients with synchronous bilateral tumors were excluded.

Information about patient, clinical, and treatment factors were collected from the electronic medical record for analysis. This included information regarding age, tumor histology, hormonal status, chemotherapy use, radiation dose and fractionation, and number of nodes taken during sentinel lymph node biopsy. Tumor hormone status was evaluated with immunohistochemical staining. Tumor positivity for HER-2 was defined as 3+ by immunohistochemistry or FISH ratio of greater than 2.0. Pathologic review was performed at a College of American Pathologists accredited laboratory. The primary endpoint was development of breast lymphedema documented in the clinic chart by our trained lymphedema nurses.

\section{Breast Lymphedema Monitoring}

All patients underwent surgery at our institution and were enrolled preoperatively into a lymphedema clinic run by two trained nurses (S.K and M.J.W). Baseline measurements were made pre-operatively. Follow-up appointments were made serially at $4,8,12$, 18 , and 24 months from surgery, and more frequently in symptomatic patients for monitoring. Patients found to be symptomatic on exam in the surgery, radiation, or medical oncology clinic were sometimes sent for urgent evaluation earlier than scheduled. Adequate follow-up for this study was defined as at least one follow-up visit post-surgery.

During each visit, clinical assessment was performed and development of breast and arm lymphedema were noted. Breast lymphedema was assessed as either present or absent based on physical exam findings, including pitting, edema, or erythema, and graded as either mild, moderate, or severe. Patients who developed breast lymphedema were given 
recommendations for treatment, including compression and massage, or referred to a lymphedema physical therapist as required. Data were collected regarding onset of lymphedema, lymphedema stage, date of resolution, and the presence of concomitant arm lymphedema.

\section{Statistical Analysis}

Comparisons between groups of patients who did and did not develop breast lymphedema were performed using Chi square tests. $P$ values $<0.05$ were considered significant. All statistical analyses were conducted using SAS 9.3 software (SAS Institute, Cary, NC).

\section{RESULTS}

\section{Patient and Treatment Characteristics}

Two hundred and thirty patients met all criteria for inclusion. Median follow-up after surgery was 15.3 months. The median age of the population was 62 (range 31-90). Seventeen patients were diagnosed with DCIS, all other patients were treated for invasive breast cancer. Eighty percent of patients were hormone positive. Most patients (90\%) had sentinel lymph node biopsy. Most patients without lymph node sampling were patients diagnosed with DCIS. The median number of lymph nodes removed was 3 (range 0-9). Seven patients had a positive node at sentinel lymph node biopsy; four had N1 disease, and three had N1mic disease. One hundred and eighty-nine patients $(82 \%)$ were treated with hypofractionated radiation, defined as dose $>2$ Gray per fraction, and $68 \%$ received a boost to the lumpectomy cavity. Seventy percent of patients did not receive any chemotherapy. Patient and treatment characteristics are summarized in Table 1.

\section{Breast Lymphedema Development}

Forty-three patients were diagnosed with lymphedema of the breast (18.7\%). Among

\begin{tabular}{|c|c|}
\hline \multicolumn{2}{|c|}{$\begin{array}{l}\text { TABLE } 1 \\
\text { Patient and Treatment Characteristics }\end{array}$} \\
\hline Variable & $\mathbf{N}(\%)$ \\
\hline \multicolumn{2}{|l|}{$\begin{array}{c}\text { Patient Characteristics } \\
\end{array}$} \\
\hline $\begin{array}{l}\text { Age at Diagnosis (Years) } \\
\text { Median }\end{array}$ & 62 \\
\hline Range & $31-90$ \\
\hline Invasive Breast Cancer & $213(93 \%)$ \\
\hline Histology & \\
\hline Hormone-positive & $183(80 \%)$ \\
\hline Triple Negative & $28(12 \%)$ \\
\hline HER-2+ & $19(8 \%)$ \\
\hline \multicolumn{2}{|l|}{ Size } \\
\hline T1 & $180(78 \%)$ \\
\hline $\mathrm{T} 2$ & $50(22 \%)$ \\
\hline \multicolumn{2}{|c|}{$\begin{array}{l}\text { Treatment Characteristics } \\
\text { Sentinel Lymph Node Biopsy Performed } 207 \text { (90\%) }\end{array}$} \\
\hline Lymph Nodes Sampled & \\
\hline Median & 3 \\
\hline Range & $0-9$ \\
\hline \multicolumn{2}{|l|}{ Radiation Fractionation } \\
\hline Conventional Fractionation & $41(18 \%)$ \\
\hline Hypofractionation & $189(82 \%)$ \\
\hline Use of Radiation Boost & $156(68 \%)$ \\
\hline Use of Chemotherapy & $69(30 \%)$ \\
\hline Taxane-based & $67(29 \%)$ \\
\hline Trastuzumab & $17(7.4 \%)$ \\
\hline
\end{tabular}

those who developed breast lymphedema, rates of grade 1 and 2 breast lymphedema were $93 \%$ and $7 \%$, respectively. There were no cases of severe breast lymphedema. The median time from surgery to diagnosis of breast lymphedema was 7.8 months (range 1.1 - 19.5 months). Breast lymphedema developed within 6 months of surgery in $18.6 \%$ of the patients, within 6-12 months for $58.1 \%$ of the patients, and after 12 months for the remainder. Twelve patients $(5.2 \%)$ in this population developed evidence of arm lymphedema. There were only 3 patients who developed concomitant breast and arm lymphedema. All patients who developed lymphedema were seen or referred to our lymphedema clinic for treatment. Treatment recommendations for management made in the clinic included wearing a supportive bra, use of compression wrap, manual lymphatic drainage, or a combination of these. Sixty-one percent of cases resolved by last follow-up; all patients with resolution received instruction on manual lymphatic drainage and a compression garment for the breast and chestwall. 
Median time to resolution of breast lymphedema was 3.7 months. Fifty percent of patients without resolution of breast lymphedema had less than 6 months of follow-up since onset. Only five patients $(11.6 \%)$ had persistent, unresolved lymphedema of the breast for 12 months or greater. The prevalence of breast lymphedema over time can be seen in Table 2 .

\begin{tabular}{|c|c|c|c|}
\hline \multirow{2}{*}{$\begin{array}{c}\text { Time from } \\
\text { surgery }\end{array}$} & \multirow{2}{*}{$\begin{array}{c}\text { Overall } \\
\text { Prevalence } \\
(\%)\end{array}$} & \multicolumn{2}{|c|}{ Grade } \\
\hline & & $\begin{array}{c}\text { Mild edema } \\
\text { (\%) }\end{array}$ & $\begin{array}{l}\text { Moderate } \\
\text { edema (\%) }\end{array}$ \\
\hline 0-3 months & $2.2 \%$ & $100 \%$ & $0 \%$ \\
\hline 3-6 months & $2.6 \%$ & $83.3 \%$ & $16.7 \%$ \\
\hline 6-9 months & $10 \%$ & $91.3 \%$ & $8.7 \%$ \\
\hline 9-12 months & $11.7 \%$ & $92.6 \%$ & $7.4 \%$ \\
\hline 12-18 months & $10 \%$ & $91.3 \%$ & $8.7 \%$ \\
\hline 18-24 months & $7.8 \%$ & $100 \%$ & $0 \%$ \\
\hline 24+ months & $3.9 \%$ & $100 \%$ & $0 \%$ \\
\hline
\end{tabular}

\section{Predictors of Breast Lymphedema Develop- ment}

Univariate associations between potential risk factors and development of breast lymphedema were examined and are reported in Table 3. There was no association between development of breast lymphedema and age, $T$ stage, use of hypofractionated whole breast radiation therapy, application of radiation boost, lymph node biopsy, number of lymph nodes removed, development of arm lymphedema, or use of taxane-based chemotherapy (all p>0.1). There was significance for tumor subtype $(p=0.04)$ and a trend for use of herceptin chemotherapy $(\mathrm{p}=0.09)$.

\section{DISCUSSION}

As paradigms shift toward avoiding toxicity, lumpectomy with adjuvant radiation continues to gain prominence as a key treatment for women with early-stage breast cancer. Among these patients, the incidence and risk factors for developing breast lymphedema, which can greatly affect patient satisfaction with treatment, remains an underexplored area. In this study, we examined the incidence of breast lymphedema following lumpectomy and investigated potential predictors of its incidence. Our findings demonstrate that this is a common problem, even amongst patients without extensive surgery, though one that generally resolves withconservative management.

A systematic review by Verbelen in patients undergoing breast-conserving surgery reported breast lymphedema rates ranging from $0-90.4 \%$ (5). Our rates of breast lymphedema are on the lower end of that spectrum. However, many of these studies are older, and the high range likely reflects patients treated with more aggressive surgical techniques and longer courses of radiation. Additionally, we limited our study only to patients receiving sentinel lymph node biopsy or less, and there is some evidence that more extensive axillary surgery is correlated with worse edema (6). Finally, these studies used a multitude of techniques to identify breast lymphedema, as there is no formal definition currently in practice. Our results are more in concordance with recent studies that have used physical examination to assess for breast lymphedema. Degnim et al prospectively enrolled patients undergoing BCS and noted rates of mild breast lymphedema of $29 \%$ and moderate breast lymphedema of $1.6 \%$ based on physical exam by trained lymphedema nurses (3). Kelemen et al performed a cohort study examining late side effects in patients receiving both adjuvant radiation and systemic therapy and found rates of $7 \%$ and $4 \%$ for mild and moderate breast lymphedema, respectively, based on physician assessment (7). These studies, along with ours, suggest a range of 10-30\% using current surgical techniques, and associated radiation and systemic guidelines, when assessing lymphedema using a clinical exam.

There are currently no formal guidelines for management of breast lymphedema. In our clinic, treatment recommendations were similar to those that have been shown to be 


\section{TABLE 3}

Univariate Predictors of Breast Lymphedema Development

\begin{tabular}{|c|c|c|}
\hline Variable & Odds Ratio & P-Value \\
\hline \multicolumn{3}{|l|}{ Age (Years) } \\
\hline$<50$ & Reference & \multirow{2}{*}{0.47} \\
\hline$>50$ & $0.7(0.25-1.89)$ & \\
\hline \multicolumn{3}{|l|}{ Subtype } \\
\hline Hormone-positive & Reference & \multirow{3}{*}{0.04} \\
\hline Triple Negative & $2.6(0.94-6.98)$ & \\
\hline HER-2+ & $7.6(1.4-42.0)$ & \\
\hline \multicolumn{3}{|l|}{ Size } \\
\hline T1 & Reference & \multirow{2}{*}{0.78} \\
\hline $\mathrm{T} 2$ & $1.1(0.55-2.39)$ & \\
\hline \multicolumn{3}{|l|}{ Sentinel Lymph Node Biopsy Performed } \\
\hline No & Reference & \multirow{2}{*}{0.58} \\
\hline Yes & $1.6(0.45-5.64)$ & \\
\hline Lymph Nodes Sampled (continuous) & $0.9(0.75-1.06)$ & 0.19 \\
\hline \multicolumn{3}{|l|}{ Radiation Fractionation } \\
\hline Conventional Fractionation & Reference & \multirow{2}{*}{0.78} \\
\hline Hypofractionation & $0.9(0.4-2.21)$ & \\
\hline \multicolumn{3}{|l|}{ Use of Radiation Boost } \\
\hline No & Reference & \multirow{2}{*}{0.43} \\
\hline Yes & $0.8(0.38-1.52)$ & \\
\hline \multicolumn{3}{|l|}{ Use of Chemotherapy } \\
\hline No & Reference & \multirow{2}{*}{0.68} \\
\hline Yes & $0.2(0.57-2.36)$ & \\
\hline \multicolumn{3}{|l|}{ Use of Taxane-based chemotherapy } \\
\hline No & Reference & \multirow{2}{*}{0.58} \\
\hline Yes & $1.2(0.60-2.49)$ & \\
\hline \multicolumn{3}{|l|}{ Use of Trastuzumab } \\
\hline No & Reference & \multirow{3}{*}{0.09} \\
\hline Yes & $2.6(0.90-7.46)$ & \\
\hline Yes & & \\
\hline
\end{tabular}


effective for arm lymphedema, including compression garments and massage. With these conservative measures, most patients had resolution of their physical exam findings within 6 months, compared to a natural history of breast lymphedema that suggests that patients improve around 2 years (8).

Very few patients with sufficient follow-up had persistent edema even at 1 year following surgery. This suggests that measures extrapolated from the management of arm edema, compression and massage, are effective.

Risk factors for the development of breast lymphedema are another area of controversy. Lymphedema of the breast is thought to result from disruption of normal lymphatic channels via surgery and radiation. As a result, these two modalities are often thought to be the primary drivers of lymphedema development. However, this was not borne out in our study. From a surgical perspective, neither the number of nodes removed, nor whether any axillary procedure was performed at all, was a significant predictor of lymphedema development. Although a limitation of these data is the low average number of nodes removed in our cohort compared to other studies, which could affect lymphedema development. Other studies also demonstrate that while full axillary node dissections may contribute to the development of breast lymphedema $(6,9)$, there is no difference between patients undergoing sentinel lymph node biopsy and no axillary procedure (4). This suggests that less comprehensive axillary procedures do not significantly impair breast lymphatic drainage beyond a lumpectomy alone. With regards to radiation parameters, including fractionation scheme and use of boost, we also did not find any relationship with development of breast lymphedema. Generally, hypofractionated radiation schemes have been found to be equivalent to conventionally fractionated treatments with regards to lymphedema of the breast $(10,11)$. One prominent prospective study showed less risk of breast lymphedema in patients receiving hypofactionation as compared to conventional fractionation (12). Other studies suggest that the delivery of any radiation puts the patients at higher risk for breast lymphedema development (13). This was not assessed in our current study as we did not have any patients who did not receive radiation.

Prior studies have suggested that chemotherapy may contribute to the development of arm lymphedema (13). With regards to the breast specifically, Adrianssens et al demonstrated increased rates in patients receiving chemotherapy (4). Taxanes have often been reported to be associated with lymphedema, possibly due to protein leak from capillaries (14). Our study did not show a significant increase in breast lymphedema for patients receiving taxanes. Interestingly, there was a significantly higher risk of breast lymphedema in patients with HER-2 positive disease. There was also a trend towards higher rates of breast lymphedema in patients receiving Herceptin. These are likely related, but unfortunately the small number of patients prevented multivariate analysis. One patient with HER-2 positive disease who did not receive Herceptin developed breast lymphedema, which accounts for the difference in significance between these two variables. The incidence of breast lymphedema was higher in HER-2 positive patients as compared to hormone positive and triple negative patients, who also would have received taxane based chemotherapy. It is known that the immune system is heavily involved during treatment of HER-2 positive cells with targeted therapy. Tumors with a pathologic complete response often show high levels of immune cells present in the tumor bed and gene signatures associated with immune response (15). Theoretically, the increased breast lymphedema rate may be related to immune responses related to treatment of HER-2 tumors rather than direct effects of the systemic therapy.

The lack of correlation between arm lymphedema and breast lymphedema also supports a different mechanism for development of breast lymphedema as compared to arm lymphedema. Further research is needed to further assess the mechanisms and risks 
factors for breast lymphedema development in order to guide prevention and treatment management.

Strengths of the current study include its large size in a homogeneous patient population relative to other studies that have investigated this subject. Additionally, all patients were evaluated and treated in a dedicated lymphedema clinic. However, there are several limitations of this study as well. This is a retrospective study performed at a single institution; it is possible that there are certain surgical or radiation practices at our institution that influence our results compared with other published data. Additionally, there is no objective criteria for the determination of breast lymphedema, so comparisons with other studies are difficult to make. Finally, we chose to exclude patients who underwent observation (no radiation) after lumpectomy, which may introduce selection bias. Future directions of research include attempting to standardize breast lymphedema definitions and incorporating objective measurements such as ultrasound or bioimpedance.

Additionally, treatment paradigms continue to evolve, with multiple ongoing studies investigating the elimination of adjuvant radiation or even lumpectomy in certain populations of early-stage breast cancer. Given our results, avoidance of surgery or radiation likely serves as the best option for avoiding development of breast lymphedema, and this current population may serve as a baseline as less intensive therapies are investigated.

Overall, breast lymphedema is a common sequela of breast-conservation surgery, though one that can improve with conservative management. Management recommendations extrapolated from treatment for arm lymphedema appear to be effective. Among patients who undergo lumpectomy and adjuvant radiation but do not receive a full axillary lymph node dissection, tumor subtype and possibly immune response may drive breast lymphedema development.

\section{CONFLICT OF INTEREST AND DISCLO-}

SURE

The authors declare no competing financial interests exist.

\section{REFERENCES}

1. Darby, S, P McGale, C Correa, et al: Effect of radiotherapy after breast-conserving surgery on 10-year recurrence and 15-year breast cancer death: Meta-analysis of individual patient data for 10,801 women in 17 randomised trials. Lancet 378 (2011), 1707-1716.

2. Siegel, RL, KD Miller, A Jemal: Cancer statistics, 2018. CA Can. J. Clin. 68 (2018), 7-30.

3. Degnim, AC, J Miller, TL Hoskin, et al: A prospective study of breast lymphedema: Frequency, symptoms, and quality of life. Breast Can. Res. Treat. 134 (2012), 915-922.

4. Adriaenssens, N, H Verbelen, P Lievens, et al: Lymphedema of the operated and irradiated breast in breast cancer patients following breast conserving surgery and radiotherapy. Lymphology 45 (2012), 154-164.

5. Verbelen, H, N Gebruers, $\mathrm{T}$ Beyers, et al: Breast edema in breast cancer patients following breast-conserving surgery and radiotherapy: A systematic review. Breast Can. Res. Treat. 147 (2014), 63-71.

6. Wratten, CR, PC O'Brien, CS Hamilton, et al: Breast edema in patients undergoing breast-conserving treatment for breast cancer: Assessment via high frequency ultrasound. Breast J. 13 (2007), 266-273.

7. Kelemen, G, Z Varga, G Lazar, et al: Cosmetic outcome 1-5 years after breast conservative surgery, irradiation and systemic therapy. Pathol. Oncol. Res. 18 (2012), 421-427.

8. Clarke, D, A Martinez, RS Cox, et al: Breast edema following staging axillary node dissection in patients with breast carcinoma treated by radical radiotherapy. Cancer 49 (1982), 2295-2299.

9. Ronka, RH, MS Pamilo, KA von Smitten, et al: Breast lymphedema after breast conserving treatment. Acta Oncol. 43 (2004), 551-557.

10. Chadha, M, D Vongtama, P Friedmann, et al: Comparative acute toxicity from whole breast irradiation using 3-week accelerated schedule with concomitant boost and the 6.5 -week conventional schedule with sequential boost for early-stage breast cancer. Clin. Breast Can. 12 (2012), 57-62.

11. Marcenaro, M, S Sacco, S Pentimalli, et al: Measures of late effects in conservative treatment of breast cancer with standard or hypof- 
ractionated radiotherapy. Tumori 90 (2004), 586-591.

12. Haviland, JS, JR Owen, JA Dewar, et al: The UK Standardisation of Breast Radiotherapy (START) trials of radiotherapy hypofractionation for treatment of early breast cancer: 10-year follow-up results of two randomised controlled trials. Lancet Oncol. 14 (2013), 1086-1094.

13. Senofsky, GM, FL Moffat Jr, K Davis, et al: Total axillary lymphadenectomy in the management of breast cancer. Arch. Surg. 126 (1991), 1336-1341.

14. Semb, KA, S Aamdal, P Oian: Capillary protein leak syndrome appears to explain fluid retention in cancer patients who receive docetaxel treatment. J. Clin. Oncol. 16 (1998), 3426-3432.

15. Lesurf, R, OL Griffith, M Griffith, et al:
Genomic characterization of HER2-positive breast cancer and response to neoadjuvant trastuzumab and chemotherapy-results from the ACOSOG Z1041 (Alliance) trial. Ann. Oncol. 28 (2017), 1070-1077.

Melissa Mitchell, MD, PhD

Department of Breast Radiation Oncology University of Texas

MD Anderson Cancer Center

1515 Holcombe Blvd

Houston, TX 77030

E-mail: mpmitchell@mdanderson.org

Phone: 913-980-4207 